\title{
BOUNDS FOR THE SMALL REAL AND PURELY IMAGINARY ZEROS OF BESSEL AND RELATED FUNCTIONS
}

\author{
Mourad E. H. Ismail and Martin E. Muldoon
}

\begin{abstract}
We give two distinct approaches to finding bounds, as functions of the order $\nu$, for the smallest real or purely imaginary zero of Bessel and some related functions. One approach is based on an old method due to Euler, Rayleigh, and others for evaluating the real zeros of the Bessel function $J_{\nu}(x)$ when $\nu>-1$. Here, among other things, we extend this method to get bounds for the two purely imaginary zeros which arise in the case $-2<\nu<-1$. If we use the notation $j_{\nu 1}$ for the smallest positive zero, which approaches 0 as $\nu \rightarrow-1^{+}$, we can think of $j_{\nu 1}^{2}$ as continued to $-2<\nu<-1$, where it has negative values. We find an infinite sequence of successively improving upper and lower bounds for $j_{\nu 1}^{2}$ in this interval. Some of the weakest, but simplest, lower bounds in this sequence are given by $4(\nu+1)$ and $2^{5 / 3}(\nu+1)[(\nu+2)(\nu+3)]^{1 / 3}$ while a simple upper bound is $4(\nu+1)(\nu+2)^{1 / 2}$. The second method is based on the representation of Bessel functions as limits of Lommel polynomials. In this case, the bounds for the zeros are roots of polynomials whose coefficients are functions of $\nu$. The earliest bounds found by this method already are quite sharp. Some are known in the literature though they are usually found by ad hoc methods. The same ideas are applied to get bounds for purely imaginary zeros of other functions such as $J_{\nu}^{\prime}(x), J_{\nu}^{\prime \prime}(x)$, and $\alpha J_{\nu}(x)+x J_{\nu}^{\prime}(x)$.
\end{abstract}

\section{Introduction}

The Bessel function

$$
J_{\nu}(z)=\sum_{n=0}^{\infty} \frac{(-1)^{n}(z / 2)^{2 n+\nu}}{n ! \Gamma(\nu+n+1)}
$$

has all its zeros real for $\nu>-1$. For $-2<\nu<-1$, a theorem of Hurwitz (see [11], [37, p. 483]) shows that two purely imaginary zeros appear. Although a great deal of attention has been paid to finding bounds, monotonicity properties, and approximations for the real zeros, there are fewer results on the purely imaginary zeros. Here we give two systematic approaches to finding bounds for the smallest real or purely imaginary zeros of $J_{\nu}(z)$ and related functions. Our techniques give sequences of bounds which generalize many isolated results in the literature; see, e.g., [13], [14], [15], [16], and [17].

Our first approach is based on an old general method for finding inequalities for positive roots of functions represented by power series. Watson [37, pp. 500-501] attributes it to Euler in the case of zeros of the Bessel function of order zero and further developments are due to Encke [10], Cayley [5], Rayleigh [33], Lamb [25], and

Received July 9, 1993, revised April 27, 1994.

1991 Mathematics Subject Classification: 33C10.

Key words and phrases: Bessel functions, zeros, inequalities, Lommel polynomials.

Research of the first author supported by NSF Grant DMS 9203659.

Research of the second author supported by NSERC Grant A5199. 
others. See [31, pp. 65-67] for a description of some of this work and that of [1], [2], [4], [29], and [34]. The method appears to be most useful when the roots are all positive, but we will apply it also in cases where there is one negative root and the other roots are positive. In this way we get inequalities for, and discuss the variation with respect to order of, the purely imaginary roots of certain functions related to Bessel functions, in particular the function $J_{\nu}(x)$ when $-1<\nu<-2$.

Our second method of generating bounds is based on the representation of Bessel functions as appropriate limits of Lommel polynomials. This provides an obvious way in which to find approximations for the zeros. What we show here is that, under appropriate conditions, these approximations provide upper and lower bounds for the zeros. The two methods lead to different bounds, the ones coming from the Lommel approach being generally better. But the approximations given by the Euler-Rayleigh method are easier to generate, especially if symbolic algebra is used. The two methods have this in common: that one-sided bounds for the smallest zero, in the case where all the zeros are real, become alternately upper and lower bounds for the modulus of a purely imaginary zero in the case of a single pair of purely imaginary zeros (with all other zeros real).

It is important to emphasize that what we describe in $\S 3$ is a method for generating successively sharper but more complicated bounds. In the theorems of $\S \S 5-8$, we give only the first few such bounds explicitly.

\section{Variation of the zeros with $\nu$}

It is well-known $\left[37\right.$, p. 508] that the positive zeros of $J_{\nu}(z)$ increase as $\nu$ increases on their interval of definition. The general behaviour of these zeros as functions of the order is well illustrated in the diagram in [37, p. 510].

The (generally complex) zeros of the even entire function $z^{-\nu} J_{\nu}(z)$, for unrestricted real $\nu$, are located symmetrically with respect to both the real and imaginary axes in the $z$-plane. Following [37, p. 497], we denote the zeros of this function by $\pm j_{\nu 1}, \pm j_{\nu 2}, \pm j_{\nu 3}, \ldots$, where $\Re\left(j_{\nu n}\right)>0$ and $\left|\Re\left(j_{\nu 1}\right)\right| \leq\left|\Re\left(j_{\nu 2}\right)\right| \leq\left|\Re\left(j_{\nu 3}\right)\right| \leq \ldots$ If $\Re\left( \pm j_{\nu n}\right)=0$, for any value of $n$, we choose $j_{\nu n}$ to have its imaginary part positive.

It is instructive to consider the evolution of the zeros as $\nu$ decreases. If we start with a value of $\nu$ greater than -1 and allow $\nu$ to decrease through real values, we find that the zeros of $z^{-\nu} J_{\nu}(z)$ approach the origin symmetrically where a pair of them disappears (as far as the real number system is concerned) whenever $\nu$ passes through a negative integer. From the results of Hurwitz (see [37, p. 483]), and further results on the complex zeros (see [20], [21]), we may describe the situation as follows. As $\nu$ decreases through -1 , the numbers $\pm j_{\nu 1}$ approach the origin, collide, and move off along the imaginary axis. As $\nu$ is further decreased, to -2 , these zeros return to the origin, as will appear below. By this time, the zeros $\pm j_{\nu 2}$ have arrived at the origin and the resulting interaction causes all four zeros to move away from the origin into the complex plane (no longer along the real or imaginary axes). Whenever $\nu$ passes through a negative integer, a new pair of zeros arrives at the origin from along the real axis. As $\nu$ passes through an odd negative integer $-(2 s+1)$, one pair of the $4 s+2$ complex zeros becomes purely imaginary and remains so until $\nu$ reaches the next integer $-(2 s+2)$; see [11], [37, p. 483].

All of this suggests that to deal with purely imaginary zeros, it is advantageous to consider the squares of the zeros. We find then that $j_{\nu 1}^{2}$ can be continued analytically from the interval $(-1, \infty)$ to the interval $(-2, \infty)$. From our bounds it will follow that 


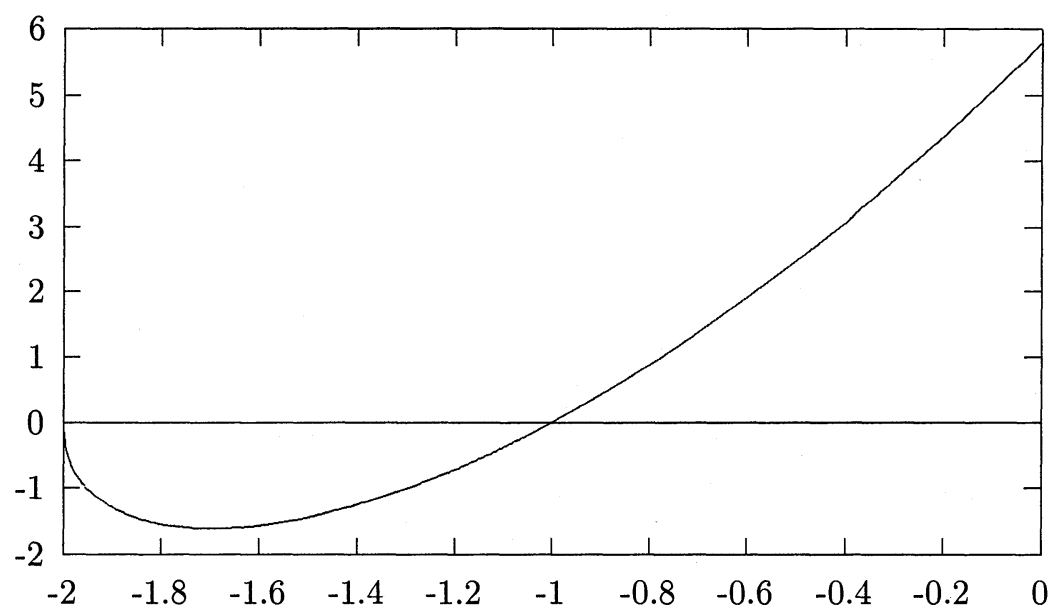

FiguRE 1. $j_{\nu 1}^{2}$ vs. $\nu$.

$j_{\nu 1}^{2}$ approaches 0 as $\nu \rightarrow-2^{+}$. In fact [23], $j_{\nu 1}^{2}$ decreases to a minimum and then increases again (to 0 ) as $\nu$ increases from -2 to -1 . See Figure 1 .

We conjecture that $j_{\nu 1}^{2}$ is convex for $-2<\nu<\infty$; cf. [8] where the convexity is proved for $0<\nu<\infty$ and conjectured for $-1<\nu<\infty$.

\section{The Euler-Rayleigh method}

Suppose that an entire function $f(z)$ has the power series expansion

$$
f(z)=1+\sum_{k=1}^{\infty} a_{k} z^{k}
$$

and an infinite product representation

$$
f(z)=\prod_{k=1}^{\infty}\left(1-\frac{z}{z_{k}}\right)
$$

where it is assumed that $\sum\left|z_{k}\right|^{-1}<\infty$. Then the following procedure can be used to express the power sums

$$
S_{m}=\sum_{k=1}^{\infty} z_{k}^{-m}
$$

in terms of the coefficients $a_{k}$. Logarithmic differentiation of (3.2) leads to

$$
\frac{f^{\prime}(z)}{f(z)}=\sum_{k=1}^{\infty} \frac{1}{z-z_{k}}
$$

whence

$$
S_{1}=-\frac{f^{\prime}(0)}{f(0)}=-a_{1} .
$$


Further differentiation of (3.3) leads to

$$
\frac{f^{\prime \prime}(z)}{f(z)}=\left(\frac{f^{\prime}(z)}{f(z)}\right)^{2}-\sum_{k=1}^{\infty} \frac{1}{\left(z-z_{k}\right)^{2}}
$$

so we obtain

$$
S_{2}=-2 a_{2}-a_{1} S_{1}=-2 a_{2}+a_{1}^{2} .
$$

This process can be repeated. The general result is

$$
S_{n}=-n a_{n}-\sum_{i=1}^{n-1} a_{i} S_{n-i}
$$

The result (3.7) can also be obtained, as outlined in [37, pp. 500-501], by comparing the coefficients of $z$ on both sides of

$$
f^{\prime}(z)=-f(z) \sum_{m=0}^{\infty} S_{m+1} z^{m},|z|<z_{1} .
$$

Formula (3.7) is actually a generalization to entire functions of Newton's formula for sums of powers of roots of a polynomial in terms of symmetric functions of the roots (or coefficients of the polynomial); see [6, pp. 134-136]. If the zeros satisfy

$$
0<\left|z_{1}\right|<\left|z_{2}\right| \leq\left|z_{3}\right| \leq \ldots
$$

we have

$$
z_{1}=\lim _{n \rightarrow \infty} \frac{S_{n}}{S_{n+1}}
$$

This is equivalent to the statement

$$
\lim _{n \rightarrow \infty} \frac{1+\left[z_{1} / z_{2}\right]^{n}+\left[z_{1} / z_{3}\right]^{n}+\ldots}{1+\left[z_{1} / z_{2}\right]^{n+1}+\left[z_{1} / z_{3}\right]^{n+1}+\ldots}=1
$$

which follows from

$$
\lim _{n \rightarrow \infty}\left(\left|\frac{z_{1}}{z_{2}}\right|^{n}+\left|\frac{z_{1}}{z_{3}}\right|^{n}+\ldots\right)=0 .
$$

This, in turn, follows from the following lemma.

Lemma 3.1. Let $1>y_{1} \geq y_{2} \geq y_{2} \geq \cdots \geq 0$ and suppose that $\sum y_{k}<\infty$. Then $\lim _{r \rightarrow \infty} \sum y_{k}^{r}=0$.

Proof. Let $\varepsilon(>0)$ be given. There exists an $m$, independent of $r$ such that $y_{m+1}+\ldots<$ $\varepsilon / 2$. Hence, for all $r \geq 1, y_{m+1}^{r}+\ldots<\varepsilon / 2$. Also, for $r>(\ln \varepsilon-\ln (2 m)) / \ln \left(y_{1}\right)$, we have $y_{1}^{r}+\cdots+y_{m}^{r}<\varepsilon / 2$, so for such $r$, we have $\sum y_{k}^{r}<\varepsilon$ and the lemma is proved.

The relation between $x_{1}$ and the sums $S_{n}$ can be made much more specific in the case where the zeros are real and positive. 
Lemma 3.2. With the notation of this section, suppose that

$$
0<x_{1}<x_{2}<\cdots
$$

Then

$$
S_{m}^{-1 / m}<x_{1}<S_{m} / S_{m+1}, \quad m=1,2, \ldots
$$

where the lower bounds increase and the upper bounds decrease to $x_{1}$ as $m \rightarrow \infty$.

Proof. The inequalities (3.12) follow easily from the definition of the $S_{n}$. That the lower bounds increase can be seen by using $S_{m}^{-1 / m}<S_{m} / S_{m+1}$. That the upper bounds decrease is a consequence of the Cauchy-Schwarz inequality.

Next we turn to a situation where all except one of the zeros is positive, the remaining one being negative.

Lemma 3.3. With the notation of this section, suppose that

$$
x_{1}<0<x_{2}<x_{3}<\ldots
$$

and that $S_{1}<0$. Then

$$
-\left|S_{2 m-1}\right|^{-1 /(2 m-1)}<x_{1}<-S_{2 m}^{-1 /(2 m)}, \quad m=1,2,3, \ldots,
$$

and

$$
S_{2 m} / S_{2 m+1}<x_{1}<S_{2 m-1} / S_{2 m}, \quad m=1,2,3, \ldots .
$$

Proof. First of all we remark that the condition $S_{1}<0$ implies that $\left|x_{1}\right|<x_{2}$ and that $S_{1}<0$ implies that $S_{m}<0$ for all odd $m$. To see this, we use the fact that $S_{1}<0$ implies

$$
-\frac{1}{x_{1}}>\frac{1}{x_{2}}+\frac{1}{x_{3}}+\cdots
$$

and hence for $m$ odd,

$$
-\frac{1}{x_{1}^{m}}>\left(\frac{1}{x_{2}}+\frac{1}{x_{3}}+\cdots\right)^{m}>\frac{1}{x_{2}^{m}}+\frac{1}{x_{3}^{m}}+\cdots
$$

or $S_{m}<0$. For $m$ even, since $S_{m}>0$, we get, by applying Lemma 3.2 to the case where $x_{1}$ is replaced by $-x_{1}$,

$$
x_{1}<-S_{m}^{-1 / m}, \quad m=2,4,6, \ldots .
$$

In case $m$ is $o d d$, it makes a difference whether $S_{m}$ is positive or negative. For $S_{m}>0$, we would not get useful information, while for $S_{m}<0$, we have

$$
0>x_{1}>-\left|S_{m}\right|^{-1 / m}, \quad m=1,3,5, \ldots
$$

We can combine the above results in the formula (3.14). The inequalities (3.15) follow easily from the definition of $S_{n}$. 
Remark. For each $m$, the upper bound given by (3.15) is weaker than that given by (3.14). This can be seen by using the consequence

$$
\left|S_{2 m-1}\right|^{1 /(2 m-1)}<S_{2 m}^{1 /(2 m)}
$$

of (3.14). Nevertheless, in our applications, the approximations given by (3.15) are simpler than those given by (3.14) - typically rational, rather than nonrational, functions of a parameter $\nu$, so we give them explicitly in the theorems of $\S \S 5-8$.

\section{Lommel polynomials}

The Lommel polynomials arise from the expression of $J_{\nu+m}(z)$ linearly in terms of $J_{\nu}(z)$ and $J_{\nu-1}(z)[37$, p. 294]:

$$
J_{\nu+m}(z)=J_{\nu}(z) R_{m, \nu}(z)-J_{\nu-1}(z) R_{m-1, \nu+1}(z) .
$$

$R_{m, \nu}(z)$ is a polynomial of degree $m$ in $1 / z$. In fact [37, p. 299],

$$
R_{0, \nu}(z)=1, \quad R_{1, \nu}(z)=\frac{2 \nu}{z}, \quad R_{2, \nu}(z)=\frac{4 \nu(\nu+1)}{z^{2}}-1,
$$

and, in general

$$
R_{m-1, \nu}(z)+R_{m+1, \nu}(z)=\frac{2(\nu+m)}{z} R_{m, \nu}(z)
$$

We have

$$
\frac{z R_{m+1, \nu}(z)}{R_{m, \nu+1}(z)}=2 \nu-\frac{z^{2}}{2(\nu+1)-\frac{z^{2}}{2(\nu+2)-\ddots}-\frac{z^{2}}{2(\nu+m)}}
$$

so that $z R_{m+1, \nu}(z) / R_{m, \nu+1}(z)$ is a convergent of the continued fraction [37, p. 303]

$$
\frac{z J_{\nu-1}(z)}{J_{\nu}(z)}=2 \nu-\frac{z^{2}}{2(\nu+1)-\frac{z^{2}}{2(\nu+2)-\cdot}-\frac{z^{2}}{2(\nu+m)-\cdot}}
$$

which follows from

$$
\frac{z J_{\nu-1}(z)}{J_{\nu}(z)}=2 \nu-\frac{z^{2}}{2(\nu+1)-\frac{z^{2}}{2(\nu+2)-\ddots}-\frac{z^{2}}{2(\nu+m)-\frac{z J_{\nu+m+1}(z)}{J_{\nu+m}(z)}}} .
$$

The following inequalities will be useful.

Lemma 4.1. For $\nu>-1$ and $0<x<j_{\nu 1}$, we have

$$
\frac{x J_{\nu-1}(x)}{J_{\nu}(x)}<\frac{x R_{m+1, \nu}(x)}{R_{m, \nu+1}(x)}, \quad m=1,2, \ldots
$$


Proof. The assertion of the lemma is, by (4.1), equivalent to the inequality

$$
\frac{x J_{\nu+m+1}(x)}{J_{\nu}(x) R_{m, \nu+1}(x)}>0, \quad 0<x<j_{\nu 1}
$$

The numerator here is clearly positive since $J_{\nu+m+1}(x)>0,0<x<j_{\nu 1}$. The positivity of the denominator follows from $R_{m, \nu+1}(x) \rightarrow+\infty, x \rightarrow 0^{+}$and from the fact that all the positive zeros of $R_{m, \nu+1}(x)$ exceed $j_{\nu 1}$. To see this, we use the fact that the Lommel polynomials $\left\{h_{n, \nu+1}(x)\right\}=\left\{R_{n, \nu+1}(1 / x)\right\}$ are orthogonal with respect to a discrete measure whose masses are supported at $\pm 1 / j_{\nu, n}, n=1,2, \ldots$ [35]. This is the approach taken in [18, pp. 197-198]. In the case where $z$ is replaced by $i y,(4.2)$ and (4.4) become

$$
\frac{i y R_{m+1, \nu}(i y)}{R_{m, \nu+1}(i y)}=2 \nu+\frac{y^{2}}{2(\nu+1)+\frac{y^{2}}{2(\nu+2)+\cdot+\frac{y^{2}}{2(\nu+m)}}}
$$

and

$$
\frac{y I_{\nu-1}(y)}{I_{\nu}(y)}=2 \nu+\frac{y^{2}}{2(\nu+1)+\frac{y^{2}}{2(\nu+2)+\ddots}+\frac{y^{2}}{2(\nu+m)+\frac{y I_{\nu+m+1}(y)}{I_{\nu+m}(y)}}} .
$$

These, together with the fact that $I_{\nu}(x)>0, \nu>-1, x>0$, lead to the following lemma.

Lemma 4.2. For $\nu>-2$ and $y>0$, we have

$$
(-1)^{m}\left[\frac{I_{\nu-1}(y)}{I_{\nu}(y)}-\frac{i R_{m+1, \nu}(i y)}{R_{m, \nu+1}(i y)}\right]>0, \quad m=1,2, \ldots
$$

It follows from (4.6) that, for $\nu>-1$, iy $R_{m+1, \nu}(i y) / R_{m, \nu+1}(i y)$ increases from $2 \nu$ (to $+\infty$, in case $m$ is odd, and to the positive value $(m+2)(\nu+m / 2)$, in case $m$ is even) as $y$ increases from 0 to $\infty$. Thus

Lemma 4.3. (i) For $-1<\nu<0$, the function iy $R_{m+1, \nu}($ iy $) / R_{m, \nu+1}($ iy $)$ has exactly one zero on $0<y<\infty$.

(ii) For $-1<\nu<-\alpha$, the function iy $R_{m+1, \nu}(i y)+(\alpha-\nu) R_{m, \nu+1}(i y)$ has exactly one zero on $0<y<\infty$ for $m$ odd and when $m$ is even, it has one zero or no zero there according as $\nu-\alpha$ is $<$ or $\geq(m+2)(\nu+m / 2)$. 


\section{Application to Bessel functions}

To apply the preceding considerations to the zeros of Bessel functions, we take

$$
f(z)=\Gamma(\nu+1) 2^{\nu} z^{-\nu / 2} J_{\nu}\left(z^{1 / 2}\right),
$$

where we use that branch of the square root function which is positive for $z$ positive. We have $z_{n}=j_{\nu n}^{2}$ and

$$
a_{n}=\frac{(-1)^{n}}{2^{2 n} n !(\nu+1) \cdots(\nu+n)} .
$$

When $\nu>-1$, all the $z_{n}$ are positive and (3.12) leads to Rayleigh's inequalities for $j_{\nu 1}^{2}[37$, p. 502]; some of these are listed explicitly in $[18,(6.7)$ to $(6.11)]$. The first two pairs are

$$
\begin{gathered}
4(\nu+1)<j_{\nu 1}^{2}<4(\nu+1)(\nu+2), \quad \nu>-1, \\
4(\nu+1)(\nu+2)^{1 / 2}<j_{\nu 1}^{2}<2(\nu+1)(\nu+3), \quad \nu>-1 .
\end{gathered}
$$

Even the first of these makes it clear that $j_{\nu 1}^{2} \rightarrow 0$ as $\nu \rightarrow-1^{+}$. In the present (Bessel function) case, there are methods of finding the successive $S_{n}$ recursively, without having to use the formula (3.7). Kishore [22] has shown that

$$
S_{n}(\nu)=\frac{1}{\nu+n} \sum_{k=1}^{n-1} S_{k}(\nu) S_{n-k}(\nu),
$$

and that

$$
S_{n}(\nu)=\frac{1}{\nu+1} \sum_{k=1}^{n-1} S_{k}(\nu+1) S_{n-k}(\nu),
$$

so that the $S_{n}(\nu)$ may be found successively, starting from

$$
S_{1}=1 /[4(\nu+1)] \text {. }
$$

The next few $S$ 's in order are

$$
S_{2}=\frac{1}{16(\nu+1)^{2}(\nu+2)}, \quad S_{3}=\frac{1}{32(\nu+1)^{3}(\nu+2)(\nu+3)} .
$$

In case $-2<\nu<-1$, we have the situation described in (3.13), i.e., $j_{\nu 1}^{2}<0<j_{\nu 2}^{2}<$ $j_{\nu 3}^{2}<\cdots$, since the notational convention described in the Introduction makes $j_{\nu 1}$ the purely imaginary zero with positive imaginary part. Also, $S_{1}<0$, so we may use (3.14) and (3.15) to generate infinite sequences of bounds. The simplest of these, got by taking $m=1,2$ in (3.14), and $m=1$ in (3.15) are given by the following theorem.

Theorem 5.1. Let $-2<\nu<-1$. Then

$$
\begin{gathered}
4(\nu+1)<j_{\nu 1}^{2}<4(\nu+1)(\nu+2)^{1 / 2}, \\
2(\nu+1)(\nu+3)<j_{\nu 1}^{2}<4(\nu+1)(\nu+2),
\end{gathered}
$$

and

$$
j_{\nu 1}^{2}>2^{5 / 3}(\nu+1)[(\nu+2)(\nu+3)]^{1 / 3}
$$


We note that the upper bound in (5.7) is the same as the lower bound in (5.4), but, of course, it is positive in (5.4) and negative in (5.7). The upper bound in (5.7) and the lower bound in (5.9) show that $j_{\nu 1}^{2}$ approaches 0 from below both as $x \rightarrow-1^{-}$ and as $x \rightarrow-2^{+}$. Numerical evidence based on further bounds of this kind indicates that $j_{\nu 1}^{2}$ decreases from 0 to $-1.60748 \ldots$ as $\nu$ increases from -2 to about -1.697 , and then increases to 0 as $\nu$ increases to -1 . (The unimodality of $-j_{\nu 1}^{2}$ on $(-2,-1)$ is proved in [23]. See Figure 1. Numerical evidence indicates also that $j_{\nu 1}^{2} /(\nu+1)$ increases from 0 to $\infty$ as $\nu$ increases from -2 to $\infty$ (we know that it increases on $(-1, \infty)\left[18\right.$, Theorem 2]) and that $j_{\nu 1}^{2} /\left[(\nu+1)(\nu+2)^{1 / 2}\right]$ decreases from about 5.48 to 4 as $\nu$ increases from -2 to -1 and then increases to $\infty$ as $\nu$ increases to $\infty$.

Now we consider bounds generated by the relation between Bessel functions and Lommel polynomials. The case $\nu>-1$ has been dealt with partially in [18, p. 198]; the important point (following from Lemma 4.1 ) is that the largest zero of $h_{n, \nu+1}(x)=$ $R_{n, \nu+1}(1 / x)$ provides a lower bound for $1 / j_{\nu 1}$. This leads to a sequence of upper bounds for $j_{\nu 1}^{2}$.

Theorem 5.2. Let $\nu>-1$ and let $x_{n}$ be the smallest positive zero of $R_{n, \nu+1}$. Then

$$
j_{\nu 1}^{2}<x_{n}^{2}, \quad n=2,3, \ldots
$$

In particular, for $n=2,3$, using the explicit representations

$$
\begin{gathered}
R_{2, \nu}(z)=\frac{4 \nu(\nu+1)}{z^{2}}-1 \\
R_{3, \nu}(z)=\frac{4 \nu(\nu+1)}{z}\left[\frac{2(\nu+2)}{z^{2}}-\frac{1}{\nu}\right],
\end{gathered}
$$

Theorem 5.2 gives the inequalities

$$
\begin{aligned}
& j_{\nu 1}^{2}<4(\nu+1)(\nu+2), \quad \nu>-1, \\
& j_{\nu 1}^{2}<2(\nu+1)(\nu+3), \quad \nu>-1 .
\end{aligned}
$$

In case $-2<\nu<-1$, we find

$$
2(\nu+1)(\nu+3)<j_{\nu 1}^{2}<4(\nu+1)(\nu+2)
$$

i.e, we get the same bounds except that they are now (because of Lemma 4.2) alternately upper and lower bounds for $j_{\nu 1}^{2}$. The general result is the following.

Theorem 5.3. Let $-2<\nu<-1$ and let $x_{n}$ be the positive zero of $R_{n, \nu+1}(i y)$. Then

$$
-x_{2 n-1}^{2}<j_{\nu 1}^{2}<-x_{2 n}^{2}, \quad n=1,2, \ldots
$$

Proof. It is clear from Lemma 4.2 that the single real zero of $I_{\nu}(x)$ is greater than or less than the unique positive zero of $R_{m+1, \nu+1}(i x)$ (guaranteed by Lemma 4.3) according as $m$ is odd or even.

The following table presents a comparison of the approximations provided by (3.15), (3.14), and (5.13) for the negative square of the purely imaginary zero of $J_{-1.4}(x)$. 


\begin{tabular}{|c|c|c||c|}
\hline$m$ & $(3.15)$ & $(3.14)$ & $(5.13)$ \\
\hline \hline 2 & 0.4034000208 & 1.264911064 & 0.9797958971 \\
3 & 1.131370850 & 1.113263074 & 1.131370850 \\
4 & 1.117139204 & 1.119266566 & 1.118413491 \\
5 & 1.119005770 & 1.118734346 & 1.118789993 \\
6 & 1.118752937 & 1.118788625 & 1.118783206 \\
7 & 1.118787431 & 1.118782677 & 1.118783286 \\
8 & 1.118782718 & 1.118783356 & 1.118783285 \\
9 & 1.118783362 & 1.118783277 & 1.118783285 \\
10 & 1.118783274 & 1.118783286 & 1.118783285 \\
\hline
\end{tabular}

It is clear that, once $m$ is moderately large, the roots of the algebraic equations arising in (5.13) give the best approximations for the zero. Next come the algebraic approximations provided by (3.14), while the rational approximations (3.15) are the slowest to converge to $-j_{-1.4,1}^{2}$.

5.1. Analyticity of the square of a zero. For completeness, we show here that $j_{\nu 1}^{2}$ is definable as an analytic function of $\nu$ for $-2<\nu<\infty$. From general considerations ([37, p. 507], [7]), the only point of possible difficulty is $\nu=-1$ where the origin becomes a zero. We follow the method which Elbert [7] used to show that $j_{\nu k}$ is analytic on $(-k, \infty)$. We use the notation

$$
g(\nu, z)=(\nu+1) f(z)=(\nu+1)-\frac{1}{4} z+\frac{1}{32(\nu+2)} z^{2}-\cdots
$$

Since $g(-1,0)=0$ and $g_{\nu}(-1,0)=1 \neq 0$, the implicit function theorem shows that we may solve the equation $g(\nu, z)=0$ for $\nu$ in a neighbourhood of $z=0$ in the form

$$
\nu+1=\sum_{m=1}^{\infty} c_{m} z^{m} .
$$

From the expansion

$$
\nu+1=g(\nu, z)+\frac{z}{4}\left[1-\frac{z}{8(\nu+2)}+\cdots\right],
$$

we see that the $c$ 's are real and, using an expansion due to R. Piessens [28, (9)], that $c_{1}=1 / 4$. We then may use the Lagrange inversion formula to expand $z$ in the form

$$
z=4(\nu+1)+\sum_{m=2}^{\infty} d_{n}(\nu+1)^{n}
$$

in a neighbourhood of $\nu=-1$. Since [7] the first positive zero of $J_{\nu}(z)$ is the only positive zero to vanish as $\nu \rightarrow-1^{+}$, we see that the $z$ just found must coincide with $j_{\nu 1}^{2}$ in a neighbourhood of $\nu=-1$. Thus $j_{\nu 1}^{2}$ is analytic on $-2<\nu<\infty$.

The use of (5.5) and (5.6), in conjunction with the limit relation (3.9) and other results of this section, affords an efficient method of evaluating $j_{\nu 1}$ when $\nu$ is close to -1 . In Figure 1, we present a graph of $j_{\nu 1}^{2}$ versus $\nu$ based on such calculations. This figure leads to the conjecture mentioned at the end of $\S 2$. 


\section{Combinations of the Bessel function and its derivative}

Here we consider the Dini function [37, Ch. 18]

$$
M_{\nu}(z)=\alpha J_{\nu}(z)+z J_{\nu}^{\prime}(z) .
$$

The zeros of this function have been dealt with by a number of authors; see [14], [17], [36]. The zeros of a more general function, with $J_{\nu}$ replaced by a general cylinder function, have been considered in [9].

In case $\alpha+\nu \neq 0$, it is convenient to deal with the function

$$
f(z)=(\nu+\alpha)^{-1} \Gamma(\nu+1) 2^{\nu} z^{-\nu / 2} M_{\nu}\left(z^{1 / 2}\right) .
$$

We write $z_{n}$ for the zeros of this function ordered by increasing real part. Equation (3.1) holds with

$$
a_{n}=\frac{(-1)^{n}(\alpha+2 n+\nu)}{(\nu+\alpha) 2^{2 n} n !(\nu+1) \cdots(\nu+n)}, \quad n=1,2, \ldots
$$

We have

$$
\begin{gathered}
S_{1}=\frac{\alpha+\nu+2}{4(\nu+1)(\nu+\alpha)}, \\
S_{2}=\frac{\nu^{2}+(2 \alpha+8) \nu+\alpha^{2}+4 \alpha+8}{16(\nu+\alpha)^{2}(\nu+1)^{2}(\nu+2)}, \\
S_{3}=\frac{(\alpha+\nu)^{3}+6 \alpha^{2}+16 \nu^{2}+18 \alpha \nu+18 \alpha+38 \nu+24}{32(\nu+\alpha)^{3}(\nu+1)^{3}(\nu+2)(\nu+3)},
\end{gathered}
$$

etc. It is known [37, p. 597] that in case $\alpha+\nu>0, \nu>-1$, the function $\alpha J_{\nu}(z)+z J_{\nu}^{\prime}(z)$ has only real zeros, so Lemma 3.2 shows that for the first positive zero we have a sequence of bounds, the first few of which are given by the following theorem.

Theorem 6.1. Let $\alpha+\nu>0, \nu>-1$, and let $x_{1}$ be the smallest positive zero of the function $\alpha J_{\nu}(z)+z J_{\nu}^{\prime}(z)$. Then we have the lower bounds

$$
\begin{gathered}
x_{1}^{2}>\frac{4(\nu+\alpha)(\nu+1)}{\alpha+\nu+2}, \\
x_{1}^{2}>\frac{4(\nu+\alpha)(\nu+1)(\nu+2)^{1 / 2}}{\left(\alpha^{2}+\nu^{2}+4 \alpha+8 \nu+2 \alpha \nu+8\right)^{1 / 2}}, \\
x_{1}^{2}>\frac{2^{5 / 3}(\nu+\alpha)(\nu+1)(\nu+2)^{1 / 3}(\nu+3)^{1 / 3}}{\left((\alpha+\nu)^{3}+6 \alpha^{2}+16 \nu^{2}+18 \alpha \nu+18 \alpha+38 \nu+24\right)^{1 / 3}},
\end{gathered}
$$

and the upper bounds

$$
x_{1}^{2}<\frac{4(\alpha+\nu+2)(\nu+\alpha)(\nu+1)(\nu+2)}{(\alpha+\nu)^{2}+4 \alpha+8 \nu+8},
$$

and

$$
x_{1}^{2}<\frac{2\left((\alpha+\nu)^{2}+4 \alpha+8 \nu+8\right)(\nu+\alpha)(\nu+1)(\nu+3)}{(\alpha+\nu)^{3}+6 \alpha^{2}+16 \nu^{2}+18 \alpha \nu+18 \alpha+38 \nu+24} .
$$


Inequality (6.10), in conjunction with (5.10), shows that under the hypotheses of Theorem $6.1, x_{1}^{2}<j_{\nu 1}^{2}$.

Using the recurrence relation

$$
z J_{\nu}^{\prime}(z)+\nu J_{\nu}(z)=z J_{\nu-1}(z)
$$

we get

$$
\alpha J_{\nu}(x)+z J_{\nu}^{\prime}(x)=J_{\nu}(x)\left[\alpha-\nu+\frac{x J_{\nu-1}(x)}{J_{\nu}(x)}\right],
$$

so using Lemma 4.1, we get

$$
\alpha J_{\nu}(x)+z J_{\nu}^{\prime}(x)<J_{\nu}(x)\left[\alpha-\nu+\frac{x R_{m+1, \nu}(x)}{R_{m, \nu+1}(x)}\right]
$$

for $\nu>-1,0<x<j_{\nu 1}$. This leads to the following result.

Theorem 6.2. Under the hypotheses of Theorem 6.1, we have $x_{1}^{2}<z_{m}^{2}, m=1,2, \ldots$, where $z_{m}$ is the smallest positive zero of $x R_{m+1, \nu}(x)+(\alpha-\nu) R_{m, \nu+1}(x)$.

In particular, this gives, for $m=1,2$,

$$
\begin{aligned}
& x_{1}^{2}<2(\nu+1)(\nu+\alpha), \\
& x_{1}^{2}<\frac{4(\nu+1)(\nu+2)(\nu+\alpha)}{3 \nu+\alpha+4} .
\end{aligned}
$$

The last inequality here is stronger than (6.10) in the case $\alpha>-1$.

In the important special case $\alpha=0$, Theorem 6.1 specializes to the following.

Theorem 6.3. Let $\nu>0$ and let $j_{\nu 1}^{\prime}$ be the smallest positive zero of $J_{\nu}^{\prime}(x)$. Then we have the lower bounds

$$
\begin{gathered}
{\left[j_{\nu 1}^{\prime}\right]^{2}>\frac{4 \nu(\nu+1)}{\nu+2}} \\
{\left[j_{\nu 1}^{\prime}\right]^{2}>\frac{4 \nu(\nu+1)(\nu+2)^{1 / 2}}{\left(\nu^{2}+8 \nu+8\right)^{1 / 2}}} \\
{\left[j_{\nu 1}^{\prime}\right]^{2}>\frac{2^{5 / 3} \nu(\nu+1)(\nu+2)^{1 / 3}(\nu+3)^{1 / 3}}{\left(\nu^{3}+16 \nu^{2}+38 \nu+24\right)^{1 / 3}}}
\end{gathered}
$$

and the upper bounds

$$
\left[j_{\nu 1}^{\prime}\right]^{2}<\frac{4 \nu(\nu+1)(\nu+2)^{2}}{\nu^{2}+8 \nu+8}
$$

and

$$
\left[j_{\nu 1}^{\prime}\right]^{2}<\frac{2\left(\nu^{2}+8 \nu+8\right) \nu(\nu+1)(\nu+3)}{\nu^{3}+16 \nu^{2}+38 \nu+24} .
$$

In certain cases we have a situation similar to that encountered in the case $-2<$ $\nu<-1$ for the Bessel function $J_{\nu}(z)$, i.e., we have all the zeros real and a pair of conjugate purely imaginary zeros. For example, if we have $-1<\nu<-\alpha$, then the function $\alpha J_{\nu}(z)+z J_{\nu}^{\prime}(z)$ has all its zeros real and a single pair of conjugate purely imaginary zeros [37, p. 597]. An application of Lemma 3.3 gives the following theorem. 
Theorem 6.4. Let $-1<\nu<-\alpha$, and let $\pm x_{1}= \pm i \xi$ ( $\xi$ real) be the purely imaginary zeros of the function $\alpha J_{\nu}(z)+z J_{\nu}^{\prime}(z)$. Then we have the lower bounds

$$
\begin{gathered}
x_{1}^{2}>\frac{4(\nu+\alpha)(\nu+1)}{\alpha+\nu+2}, \\
x_{1}^{2}>\frac{2^{5 / 3}(\nu+\alpha)(\nu+1)(\nu+2)^{1 / 3}(\nu+3)^{1 / 3}}{\left((\alpha+\nu)^{3}+6 \alpha^{2}+16 \nu^{2}+18 \alpha \nu+18 \alpha+38 \nu+24\right)^{1 / 3}},
\end{gathered}
$$

and

$$
x_{1}^{2}>\frac{2\left((\alpha+\nu)^{2}+4 \alpha+8 \nu+8\right)(\nu+\alpha)(\nu+1)(\nu+3)}{(\alpha+\nu)^{3}+6 \alpha^{2}+16 \nu^{2}+18 \alpha \nu+18 \alpha+38 \nu+24},
$$

and the upper bounds

$$
x_{1}^{2}<\frac{4(\alpha+\nu+2)(\nu+\alpha)(\nu+1)(\nu+2)}{(\alpha+\nu)^{2}+4 \alpha+8 \nu+8},
$$

and

$$
x_{1}^{2}<\frac{4(\nu+\alpha)(\nu+1)(\nu+2)^{1 / 2}}{\left(\alpha^{2}+\nu^{2}+4 \alpha+8 \nu+2 \alpha \nu+8\right)^{1 / 2}} .
$$

R. Spigler [36] showed, in our notation, that

$$
\alpha^{2}-\nu^{2}<x_{1}^{2}<(\nu-\alpha)^{2}, \quad(\nu \geq 1 / 2) \text {. }
$$

Remark. In [23, Theorem 4.1], a key part of the proof that $-x_{1}^{2}$ is unimodal on $(-1,-\alpha)$ (where $-1 / 2 \leq \alpha<1$ and $-1<\nu<-\alpha$ ) requires the inequality

$$
3 j_{\nu 1}^{2}+x_{1}^{2}>0, \quad-1<\nu<-\alpha \text {. }
$$

In [23] this was done by using $[19,(3.2)]$

$$
\xi^{2}<-\frac{\alpha+\nu}{2+\alpha+\nu} j_{\nu 1}^{2}
$$

But it also follows from the inequalities (5.3) and (6.20).

The purely imaginary zeros of $\alpha J_{\nu}(z)+z J_{\nu}^{\prime}(z)$ are real zeros of the function

$$
I_{\nu}(y)\left[\alpha-\nu+\frac{y I_{\nu-1}(y)}{I_{\nu}(y)}\right]
$$

which, by Lemma 4.2 , is greater or less than

$$
I_{\nu}(y)\left[\alpha-\nu+\frac{i y R_{m+1, \nu}(i y)}{R_{m, \nu+1}(i y)}\right]
$$

for $0<y<\infty$ according as $m$ is even or odd. This leads to the following result.

Theorem 6.5. Under the hypotheses of Theorem 6.4, we have

$$
-z_{2 m}^{2}<x_{1}^{2}<-z_{2 m-1}^{2}, \quad m=1,2, \ldots,
$$

where $z_{m}$ is the positive zero, if any, of $i x R_{m+1, \nu}(i x)+(\alpha-\nu) R_{m, \nu+1}(i x)$. 


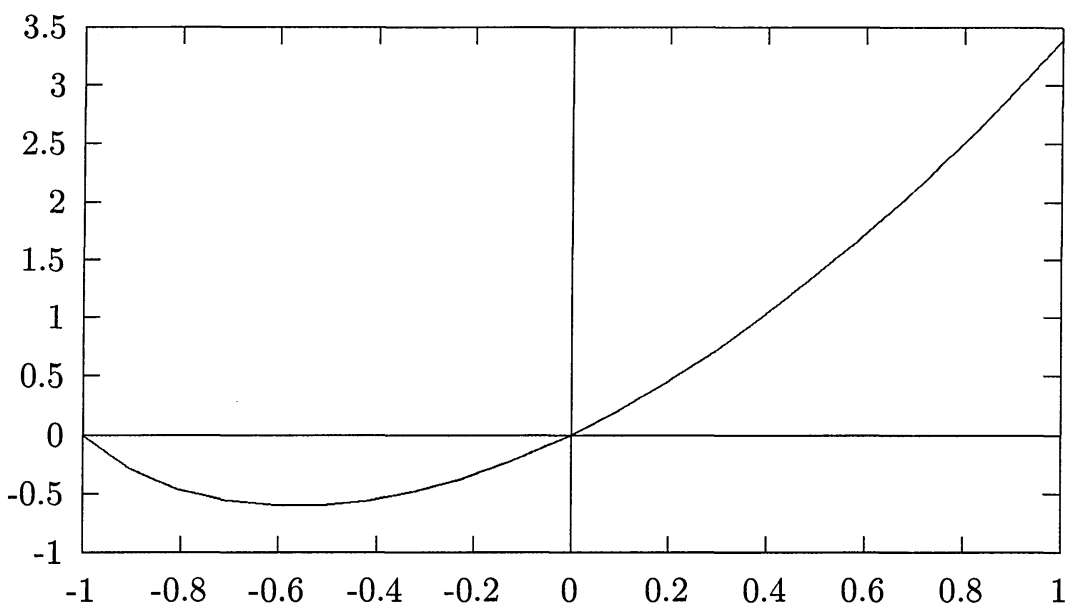

Figure $2 . j^{\prime 2}$ vi $\nu$.

Remark. Lemma 4.3 (ii) shows that such a positive zero always exists when $m$ is odd and exists under certain conditions when $m$ is even. In particular, Theorem 6.5 gives, for $m=1$,

$$
\frac{4(\nu+1)(\nu+2)(\nu+\alpha)}{3 \nu+\alpha+4}<x_{1}^{2}<2(\nu+1)(\nu+\alpha)
$$

Here we record the results of Theorem 6.4 for purely imaginary zeros of $J_{\nu}^{\prime}(z)$ in the case $-1<\nu<0$.

Theorem 6.6. Let $-1<\nu<0$ and let $\pm j_{\nu 1}^{\prime}$ denote the purely imaginary zeros of $J_{\nu}^{\prime}(z)$. We have the lower bounds

$$
\begin{aligned}
& {\left[j_{\nu 1}^{\prime}\right]^{2}>\frac{4 \nu(\nu+1)}{\nu+2}} \\
& {\left[j_{\nu 1}^{\prime}\right]^{2}>\frac{2^{5 / 3} \nu(\nu+1)(\nu+2)^{1 / 3}(\nu+3)^{1 / 3}}{\left(\nu^{3}+16 \nu^{2}+38 \nu+24\right)^{1 / 3}}} \\
& {\left[j_{\nu 1}^{\prime}\right]^{2}>\frac{2\left(\nu^{2}+8 \nu+8\right) \nu(\nu+1)(\nu+3)}{\nu^{3}+16 \nu^{2}+38 \nu+24}}
\end{aligned}
$$

and the upper bounds

$$
\left[j_{\nu 1}^{\prime}\right]^{2}<\frac{4 \nu(\nu+1)(\nu+2)^{2}}{\nu^{2}+8 \nu+8}
$$

and

$$
\left[j_{\nu 1}^{\prime}\right]^{2}<\frac{4 \nu(\nu+1)(\nu+2)^{1 / 2}}{\left(\nu^{2}+8 \nu+8\right)^{1 / 2}}
$$

Figure 2 is a graph of ${j^{\prime}}_{\nu 1}^{2}$ vs. $\nu$. 


\section{Functions involving the second derivative of a Bessel function}

Here we consider mainly the function $J_{\nu}^{\prime \prime}$ but many of our results will be valid for the more general function

$$
N_{\nu}(z)=a z^{2} J_{\nu}^{\prime \prime}(z)+b z J_{\nu}^{\prime}(z)+c J_{\nu}(z)
$$

considered by Mercer [27]. Here, as in [27], $q=b-a$ and

$$
(c=0 \text { and } q \neq 0) \text { or }(c>0 \text { and } q>0) \text {. }
$$

Unlike the situation of $J_{\nu}(z)$ and $J_{\nu}^{\prime}(z)$, the question of the reality of the zeros of $J_{\nu}^{\prime \prime}(z)$ does not seem to have been studied much. Thus, while it is classical [37] that the zeros of $J_{\nu}(z)$ and $J_{\nu}^{\prime}(z)$ are real for $\nu \geq-1$ and $\nu \geq 0$, respectively, we could not find a corresponding property for $J_{\nu}^{\prime \prime}(z)$ in the literature. Even though

$$
z^{2} J_{\nu}^{\prime \prime}(z)=\left(\nu^{2}-z^{2}\right) J_{\nu}(z)-z J_{\nu}^{\prime}(z)
$$

the reality of the zeros of $J_{\nu}^{\prime \prime}(z)$ for $\nu>1$ does not appear to follow from results on the reality of the zeros of various combinations of $J_{\nu}(z)$ and $J_{\nu}^{\prime}(z)$ in, e.g., [32] and [13]. There has been recent interest in the question of the monotonicity with respect to $\nu$ of the positive zeros of $J_{\nu}^{\prime \prime}(z)$ ([26], [38], [27]) but the discussions of these authors do not exclude the possibility of the existence of non-real zeros.

Here we present an approach to this question, based on Mercer's [27] identification of the squares of the zeros as eigenvalues of a suitable boundary value problem and leading, inter alia, to the expected conclusion that the zeros of $J_{\nu}^{\prime \prime}(z)$ are real for $\nu>1$.

We begin with a result obtained by the method of [27].

Theorem 7.1. (i) For $\nu>0$, the zeros of $N_{\nu}(z)$ are either real or purely imaginary. (ii) For $\nu \geq \max \left\{0, \nu_{0}\right\}$, where $\nu_{0}$ is the largest real root of the quadratic $Q(\nu)=$ $a \nu(\nu-1)+b \nu+c$, the zeros of $N_{\nu}(z)$ are real.

(iii) For $\nu>0,\left(a \nu^{2}+q \nu+c\right) / q>0$ and $a / q<0$, the zeros of $N_{\nu}(z)$ are all real except for a single pair which are conjugate purely imaginary.

Proof. As in [27], we note that the boundary problem

$$
\left(x y^{\prime}\right)^{\prime}=\frac{\nu^{2}}{x} y-\lambda^{2} x y
$$

subject to $y(0)=0$ and

$$
a y^{\prime \prime}(1)+b y^{\prime}(1)+c y(1)=0,
$$

has solution $y=A J_{\nu}(\lambda x)$, where $A$ is an arbitrary constant and $\lambda$ is a zero of $N_{\nu}(z)$. We proceed as in $\S 3$ of [27], except that we multiply (7.3) by $\bar{y}$ (rather than $y$ ) and integrate over $(0,1)$ to get

$$
\bar{y}(1) y^{\prime}(1)-\int_{0}^{1} x\left|y^{\prime}(x)\right|^{2} d x=\nu^{2} \int_{0}^{1} x^{-1}|y(x)|^{2} d x-\lambda^{2} \int_{0}^{1} x|y(x)|^{2} d x .
$$

Now (7.4) may be written

$$
\left[a\left(\nu^{2}-\lambda^{2}\right)+c\right] y(1)+q y^{\prime}(1)=0,
$$


so the first term in $(7.5)$ is

$$
-\frac{1}{q}|y(1)|^{2}\left[a\left(\nu^{2}-\lambda^{2}\right)+c\right] .
$$

Thus we get $[27,(3.1)]$ in the form

$$
\lambda^{2} Q=\nu^{2} P+\int_{0}^{1} x\left|y^{\prime}(x)\right|^{2} d x+\frac{c}{q}|y(1)|^{2}
$$

where

$$
P=\int_{0}^{1} \frac{1}{x}|y(x)|^{2} d x+\frac{a}{q}|y(1)|^{2}
$$

and

$$
Q=\int_{0}^{1} x|y(x)|^{2} d x+\frac{a}{q}|y(1)|^{2}
$$

Now for $\nu>0$, the integrals exist and the number $Q$ and all terms on the right of (7.7) are real. Also $Q \neq 0$, as shown in [27, p. 321]. Hence $\lambda^{2}$ is real, so the zeros are either real or purely imaginary.

To prove part (ii) we note that in this situation, the coefficients in the power series

$$
z^{-\nu} N_{\nu}(z)=\sum_{k=0}^{\infty} \frac{(-1)^{k} z^{2 k}}{2^{\nu+2 k} k ! \Gamma(\nu+k+1)} Q(\nu+2 k),
$$

alternate in sign so this function cannot have a purely imaginary zero. Thus all the zeros are real.

For part (iii), we let $\pm i y$ be purely imaginary zeros. From the Mittag-Leffler expansion [37]

$$
\frac{J_{\nu+1}(z)}{J_{\nu}(z)}=\sum_{n=1}^{\infty} \frac{2 z}{j_{\nu n}^{2}-z^{2}}
$$

we find that

$$
2 \sum_{k=0}^{\infty} \frac{1}{j_{\nu k}^{2}+y^{2}}=-\frac{a}{q}-\frac{a \nu^{2}+q \nu+c}{q y^{2}} .
$$

As $y^{2}$ increases from 0 to $\infty$ the left-hand side here decreases from $1 /[2(\nu+1)]$ to 0 while the right-hand side increases from $-\infty$ to the positive value $-a / q$. Hence there is exactly one pair of conjugate purely imaginary zeros.

Remark. It is of interest to point that for $\nu>-1$ and $\left(a \nu^{2}+q \nu+c\right) / q>0$, (i) can be proved using the Mittag-Leffler expansion (7.10). Let $z$ be a zero of $N_{\nu}(z)$. Then

$$
2 \sum_{k=0}^{\infty} \frac{1}{j_{\nu k}^{2}-z^{2}}=-\frac{a}{q}+\frac{a \nu^{2}+q \nu+c}{q z^{2}}
$$

or

$$
2 \sum_{k=0}^{\infty} \frac{j_{\nu k}^{2}-\bar{z}^{2}}{\left|j_{\nu k}^{2}-z^{2}\right|^{2}}=-\frac{a}{q}+\frac{a \nu^{2}+q \nu+c}{q|z|^{4}} \bar{z}^{2}
$$


Writing $\bar{z}^{2}=\xi+i \eta$, and taking imaginary parts in this last equation, we get

$$
-2 \eta \sum_{k=0}^{\infty} \frac{1}{\left|j_{\nu k}^{2}-z^{2}\right|^{2}}=\frac{a \nu^{2}+q \nu+c}{q|z|^{4}} \eta
$$

But this is impossible, unless $\eta=0$. Thus $\bar{z}^{2}$ is real and the result follows.

\section{Bounds for the smallest zero of $J_{\nu}^{\prime \prime}(z)$}

We can apply the method of $\S 3$ to the function $N_{\nu}(z)$ introduced in $\S 7$. The first $S$, obtained from (3.7), is in this case

$$
S_{1}=\frac{4 a(\nu+1)+a \nu^{2}+q \nu+2 q+c}{4(\nu+1)\left(a \nu^{2}+q \nu+c\right)},
$$

and they become progressively more complicated.

As an application, we see that when all the zeros are real, the simple inequality $x_{1}>S_{1}^{-1}$ gives

$$
x_{1}>\frac{4(\nu+1)\left(a \nu^{2}+q \nu+c\right)}{a \nu^{2}+(4 a+q) \nu+4 a+2 q+c} .
$$

In principle, we could derive many bounds by following this procedure. We content ourselves with stating the simplest bounds for the zeros of $J_{\nu}^{\prime \prime}(z)$. Here the function $f$ of $\S 3$ is given by

$$
f(z)=\frac{2^{\nu} \Gamma(\nu)}{\nu-1} x^{2-\nu} J_{\nu}^{\prime \prime}(\sqrt{z})=\sum_{k=0}^{\infty} \frac{(-1)^{k}(2 k+\nu)(2 k+\nu-1) x^{k}}{2^{2 k} k ! \nu(\nu-1)(\nu+1)_{k}}
$$

so that we are led to

$$
\begin{gathered}
S_{1}=\frac{\nu+2}{4 \nu(\nu-1)} \\
S_{2}=\frac{\nu^{3}+13 \nu^{2}+32 \nu+8}{16 \nu^{2}(\nu-1)^{2}(\nu+1)(\nu+2)}
\end{gathered}
$$

and

$$
S_{3}=\frac{\nu^{4}+27 \nu^{3}+138 \nu^{2}+134 \nu+24}{32 \nu^{3}(\nu-1)^{3}(\nu+1)(\nu+2)(\nu+3)} .
$$

We now apply Lemma 3.2 to the case when $\nu \geq 1$, and hence all the zeros are real by Theorem 7.1 (i), to get the following result.

Theorem 8.1. Let $\nu>1$ and let $j_{\nu 1}^{\prime \prime}$ be the smallest positive zero of $J_{\nu}^{\prime \prime}(x)$. Then we have the lower bounds

$$
\begin{gathered}
{\left[j_{\nu 1}^{\prime \prime}\right]^{2}>\frac{4 \nu(\nu-1)}{\nu+2}} \\
{\left[j_{\nu 1}^{\prime \prime}\right]^{2}>\frac{4 \nu(\nu-1)(\nu+1)^{1 / 2}(\nu+2)^{1 / 2}}{\left(\nu^{3}+13 \nu^{2}+32 \nu+8\right)^{1 / 2}}} \\
{\left[j_{\nu 1}^{\prime \prime}\right]^{2}>\frac{2^{5 / 3} \nu(\nu-1)(\nu+1)^{1 / 3}(\nu+2)^{1 / 3}(\nu+3)^{1 / 3}}{\left(\nu^{4}+27 \nu^{3}+138 \nu^{2}+134 \nu+24\right)^{1 / 3}}}
\end{gathered}
$$


and the upper bounds

$$
\begin{gathered}
{\left[j_{\nu 1}^{\prime \prime}\right]^{2}<\frac{4 \nu(\nu-1)(\nu+1)(\nu+2)^{2}}{\nu^{3}+13 \nu^{2}+32 \nu+8}} \\
{\left[j_{\nu 1}^{\prime \prime}\right]^{2}<\frac{2 \nu(\nu-1)(\nu+3)\left(\nu^{3}+13 \nu^{2}+32 \nu+8\right)}{\nu^{4}+27 \nu^{3}+138 \nu^{2}+134 \nu+24}}
\end{gathered}
$$

Even the inequality (8.7) is better, for $1<\nu<2$, than

$$
\left[j_{\nu 1}^{\prime \prime}\right]^{2}>\nu(\nu-1)
$$

got from the differential equation in [37, p. 487].

Using Theorem 7.1 (iii), we see that when $0<\nu<1$, the zeros of $J_{\nu}^{\prime \prime}(z)$ are all real, except for $\pm j_{\nu 1}^{\prime \prime}$ which are purely imaginary. Thus, from Lemma 3.3, we have the following results.

Theorem 8.2. Let $0<\nu<1$, and let $\pm j_{\nu 1}^{\prime \prime}$ be the purely imaginary zeros of $J_{\nu}^{\prime \prime}(x)$. Then we have the lower bounds

$$
\begin{gathered}
{\left[j_{\nu 1}^{\prime \prime}\right]^{2}>\frac{4 \nu(\nu-1)}{\nu+2}} \\
{\left[j_{\nu 1}^{\prime \prime}\right]^{2}>\frac{2^{5 / 3} \nu(\nu-1)(\nu+1)^{1 / 3}(\nu+2)^{1 / 3}(\nu+3)^{1 / 3}}{\left(\nu^{4}+27 \nu^{3}+138 \nu^{2}+134 \nu+24\right)^{1 / 3}}}
\end{gathered}
$$

and

$$
\left[j_{\nu 1}^{\prime \prime}\right]^{2}>\frac{2 \nu(\nu-1)(\nu+3)\left(\nu^{3}+13 \nu^{2}+32 \nu+8\right)}{\nu^{4}+27 \nu^{3}+138 \nu^{2}+134 \nu+24}
$$

and the upper bounds

$$
\begin{gathered}
{\left[j_{\nu 1}^{\prime \prime}\right]^{2}<\frac{4 \nu(\nu-1)(\nu+1)^{1 / 2}(\nu+2)^{1 / 2}}{\left(\nu^{3}+13 \nu^{2}+32 \nu+8\right)^{1 / 2}}} \\
{\left[j_{\nu 1}^{\prime \prime}\right]^{2}<\frac{4 \nu(\nu-1)(\nu+1)(\nu+2)^{2}}{\nu^{3}+13 \nu^{2}+32 \nu+8}}
\end{gathered}
$$

The inequalities for $\left[j_{\nu 1}^{\prime \prime}\right]^{2}$ show that this function vanishes as $\nu \rightarrow 1^{+}$. (See Figure 3.) It was shown by Ifantis and Siafarikas [15] that $\left[j_{\nu 1}^{\prime \prime}\right]^{2}$ decreases as $\nu$ increases from 0 to a certain $\nu_{0}\left(0.45<\nu_{0}<0.5\right)$ and then increases as $\nu$ increases from $\nu_{0}$ to 1. The corresponding property for $\left[j_{\nu 1}^{\prime}\right]^{2}$ on $(-1,0)$ and $j_{\nu 1}^{2}$ on $(-2,-1)$ has also been demonstrated [23].

The application of the Lommel method here leads to the following result.

Theorem 8.3. Let $0<\nu<1$. Then

$$
-x_{2 m-1}^{2}<\left[j_{\nu 1}^{\prime \prime}\right]^{2}<-x_{2 m}^{2}, \quad m=0,1,2, \ldots
$$

where $x_{m}$ is the smallest positive zero of the function

$$
\left[\nu^{2}+\nu+x^{2}\right] R_{m, \nu+1}(i x)-i x R_{m+1, \nu}(i x) .
$$




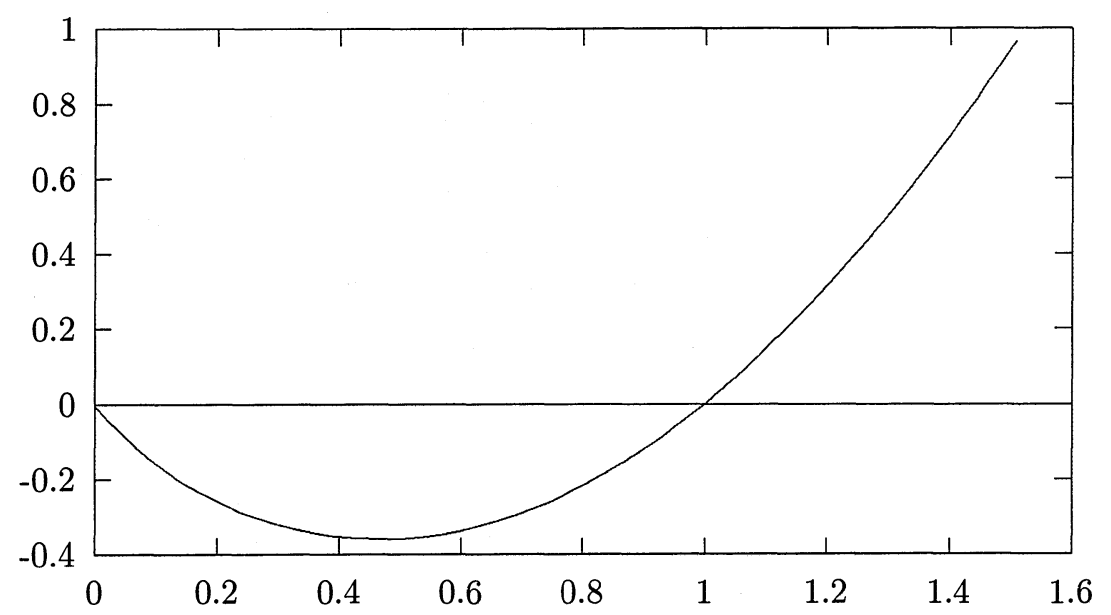

FIGURE $3 . j_{\nu 1}^{\prime \prime 2}$ vs. $\nu$.

Proof. The purely imaginary zeros of the function $J_{\nu}^{\prime \prime}(z)$ are real zeros of the function

$$
\nu^{2}+\nu+x^{2}-\frac{x I_{\nu-1}(x)}{I_{\nu}(x)}
$$

which, by Lemma 4.2 , is less than or greater than

$$
\nu^{2}+\nu+x^{2}-\frac{i x R_{m+1, \nu}(i x)}{R_{m, \nu+1}(i x)}
$$

according as $m$ is even or odd. The result follows.

The cases $m=0,1$ recover the bounds

$$
\frac{2 \nu\left(\nu^{2}-1\right)}{2 \nu+1}<\left[j_{\nu 1}^{\prime \prime}\right]^{2}<\nu(\nu-1), \quad 0<\nu<1,
$$

found in [17]. Note that both bounds vanish as $\nu$ approaches 0 and 1. Figure 3 gives a graph of $j_{\nu 1}^{\prime \prime 2}$ versus $\nu$. The formulas (8.18) are but the first in an infinite sequence of bounds. The next upper bound, got from the case $m=1$ in Theorem 8.3 is

$$
\left[j_{\nu 1}^{\prime \prime}\right]^{2}<\frac{(5 \nu+4)(\nu+1)}{2}-\frac{1}{2}\left[9 \nu^{4}+58 \nu^{3}+137 \nu^{2}+104 \nu+16\right]^{1 / 2}, \quad 0<\nu<1 .
$$

\section{Application of the Euler-Rayleigh method to Lommel polynomials}

The method of $\S 3$ can be applied to polynomials, in which case it reduces to Newton's method for expressing sums of powers of roots of a polynomial in terms of symmetric functions of the roots (or coefficients of the polynomial). Here we apply this method to the zeros of the Lommel polynomial. We use the modified notation for these polynomials [37, p. 303],

$$
g_{m, \nu}(z)=\sum_{n=0}^{[m / 2]}\left(\begin{array}{c}
m-n \\
n
\end{array}\right) \frac{(-1)^{n} \Gamma(\nu+m-n+1) z^{n}}{\Gamma(\nu+n+1)}
$$

so that

$$
R_{m, \nu+1}(z)=\left(\frac{1}{2} z\right)^{-m} g_{m, \nu}\left(\frac{1}{4} z^{2}\right)
$$


We have

$$
g_{2 m, \nu}(x)=\sum_{k=0}^{m}(-1)^{k} \frac{(2 m-k) !(\nu+1)_{2 m-k}}{(2 m-2 k) ! k !(\nu+1)_{k}} x^{k} .
$$

According to results of Hurwitz [37, p. 304], for $\nu>-2$, the zeros of $g_{2 m, \nu}(z)$ are all real and they are all positive, except when $-2<\nu<-1$, in which case one of them is negative. This, of course, ties in with the limiting relation [37, p. 302]

$$
\lim _{m \rightarrow \infty} \frac{\left(\frac{1}{2} z\right)^{\nu} g_{m, \nu}\left(\frac{1}{4} z^{2}\right)}{\Gamma(\nu+m+1)}=J_{\nu}(z) .
$$

If we take

$$
f(z)=g_{2 m, \nu}(z / 4) /(\nu+1)_{2 m},
$$

we have the situation described in $\S 3$, with

$$
\begin{gathered}
a_{1}=-\frac{2 m-1}{4(\nu+1)(\nu+2 m)}, \\
a_{2}=\frac{(2 m-2)(2 m-3)}{32(\nu+1)(\nu+2)(\nu+2 m)(\nu+2 m-1)} .
\end{gathered}
$$

This gives, using Lemma 3.2 and relations (3.4) and (3.6) for $S_{1}$ and $S_{2}$,

$$
\frac{4(\nu+1)(\nu+2 m)}{2 m-1}<x_{1}<\frac{4(\nu+1)(\nu+2)(\nu+2 m)(\nu+2 m-1)(2 m-1)}{8 m^{3}+(12 \nu-4) m^{2}+\left(6 \nu^{2}-4 \nu\right) m-5 \nu^{2}-5 \nu-2},
$$

for $\nu>-1$, where $x_{1}$ is the smallest (positive) zero of $f(z)$. This reduces to (5.3) as $m \rightarrow \infty$. It is clear that we could find analogues for Lommel polynomials of all the inequalities found here, by the Euler-Rayleigh method, for zeros of Bessel and related functions.

Acknowledgment. We are indebted to Árpád Elbert and Lee Lorch for their comments on earlier versions of this paper. We thank the referees for several corrections and comments.

\section{References}

1. S. Ahmed, On the zeros of confluent hypergeometric functions, Lett. Nuovo Cimento, 25 (1979), $52-56$.

2. S. Ahmed, C. Giordano, and A. Laforgia, Approximations for the zeros of the derivative of Bessel functions, Atti Accad. Sci. Torino 118 (1984), 217-225.

3. J. R. Airey, Bessel functions of small fractional order and their application to problems of elastic stability, Phil. Mag. (6) 41 (1921), 200-205.

4. H. Buchholz, Die Summe der reziproken Potenzen der Nullstellen von $M_{\chi, \mu / 2}(z)$ hintsichlich $z$, Z. Angew. Math. Mech. 43 (1951), 149-152.

5. A. Cayley, Appendix to Rayleigh's paper [33], Proc. London Math. Soc. 5 (1874), 123-124, Collected Papers, vol. 9 (1896), 19-20.

6. L. E. Dickson, A First Course in the Theory of Equations, Wiley, New York, 1922.

7. Á. Elbert, Concavity of the zeros of Bessel functions, Studia Sci. Math. Hungar. 12 (1977), 81-88.

8. Á. Elbert and A. Laforgia, On the square of the zeros of Bessel functions, SIAM J. Math. Anal. 15 (1984), 206-212.

9. Á. Elbert and P. D. Siafarikas, On the zeros of $a C_{\nu}(x)+x C_{\nu}^{\prime}(x)$, where $C_{\nu}(x)$ is a cylinder function, J. Math. Anal. Appl. 164 (1992), 21-33.

10. J. F. Encke, Allgemeine Auflösung der numerischen Gleichungen, J. Reine Angew. Math. 22 (1841), 193-248.

11. A. Hurwitz, Ueber die Nullstellen der Bessel'schen Function, Math. Ann. 33 (1889), 246-266. 
12. E. Hille and G. Szegö, On the complex zeros of the Bessel function, Bull. Amer. Math. Soc. 49 (1943), 605-610. Reprinted in G. Szegö, Collected Papers, vol. 2, Birkhäuser, 1982, 855-861.

13. E. K. Ifantis, C. G. Kokologiannaki, and C. B. Kouris, On the positive zeros of the second derivative of Bessel functions, J. Comp. Appl. Math. 34 (1991), 21-31.

14. E. K. Ifantis and P. D. Siafarikas, Bounds for the first positive zero of a mixed Bessel function, J. Comp. Appl. Math. 21 (1988), 245-249.

15. __ A result on the imaginary zeros of $J_{\nu}^{\prime \prime}(z)$, J. Approx. Theory 62 (1990), 192-196.

16. E. K. Ifantis, P. D. Siafarikas, and C. B. Kouris, Upper bounds for the first zeros of a Bessel function, J. Comp. Appl. Math. 17 (1987), 355-358.

17. _ The imaginary zeros of a mixed Bessel function, Z. Angew. Math. Phys. 39 (1988), $157-165$.

18. M. E. H. Ismail and M. E. Muldoon, On the variation with respect to a parameter of zeros of Bessel and q-Bessel functions, J. Math. Anal. Appl. 135 (1988), 187-207.

19. __ Zeros of combinations of Bessel functions and their derivatives, Appl. Anal. 31 (1988), 73-90.

20. M. K. Kerimov and S. L. Skorokhodov, Evaluation of complex zeros of Bessel functions $J_{\nu}(z)$ and $I_{\nu}(z)$ and their derivatives, U.S.S.R. Comput. Math. and Math. Phys. 24 (1984), 131-141 (Russian original, Ž. Vyčisl. Mat. i Mat. Fiz. 24 (1984), 1497-1513).

21. Calculation of the multiple zeros of the derivatives of cylindrical Bessel functions $J_{\nu}(z)$ and $Y_{\nu}(z)$, U.S.S.R. Comput. Math. and Math. Phys. 25 (1985), 101-107 (Russian original, Ž. Vyčisl. Mat. i Mat. Fiz. 25 (1985), 1749-1760).

22. N. Kishore, The Rayleigh polynomials, Proc. Amer. Math. Soc. 14 (1963), 527-533.

23. C. G. Kokologiannaki, M. E. Muldoon, and P. D. Siafarikas, A unimodal property of purely imaginary zeros of Bessel and related functions, Canad. Math. Bull., 37 (1994), 365-373.

24. C. G. Kokologiannaki and P. D. Siafarikas, Non-existence of complex and purely imaginary zeros of a transcendental equation involving Bessel functions, Z. für Analysis 10 (1991), 563-567.

25. H. Lamb, Note on the induction of electric currents in a cylinder placed across the lines of magnetic force, Proc. London Math. Soc. 15 (1884), 270-274.

26. L. Lorch and P. Szego, On the points of inflection of Bessel functions of positive order, Canad. J. Math. 42 (1990), 933-948; ibid. 1132.

27. A. McD. Mercer, The zeros of $a z^{2} J_{\nu}^{\prime \prime}(z)+b z J_{\nu}^{\prime}(z)+c J_{\nu}^{\prime}(z)$ as a function of order, Internat. J. Math. and Math. Sci. 15 (1992), 319-322.

28. R. Piessens, A series expansion for the first positive zero of the Bessel function, Math. Comp. 42 (1984), 195-197.

29. R. Piessens and S. Ahmed, Approximations for the turning points of Bessel functions, J. Comput. Phys. 64 (1986), 253-257.

30. G. Pólya, Graeffe's method for eigenvalues, Numer. Math. 11 (1968), 315-319.

31. E. Ya. Riekstinš, Asymptotics and Approximations of Roots of Equations (Russian), Iznatne, Riga, 1991.

32. A. D. Rawlins, A new class of Bessel function inequality useful for investigating the roots of a class of transcendental equation involving Bessel functions, Proc. Roy. Soc. Edinburgh $\mathbf{7 4 A}$ (1974-75), 231-238.

33. Lord Rayleigh, Note on the numerical calculation of the roots of fluctuating functions, Proc. London Math. Soc. 5 (1874), 119-124, Scientific Papers, vol. 1 (Dover, 1964), 190-195.

34. D. K. Ross and A. Mahajan, On zeros of special functions, Internat. J. Math. Educ. Sci. Technol. 15 (1984), 202-212.

35. H. M. Schwarz, A class of continued fractions, Duke Math. J. 6 (1940), 48-65.

36. R. Spigler, Sulle radici dell'equazione $A J_{\nu}(x)+B x J_{\nu}^{\prime}(x)=0$, Atti Sem. Mat. Fis. Univ. Modena 24 (1975), 339-419.

37. G. N. Watson, A Treatise on the Theory of Bessel Functions, 2nd ed., Cambridge University Press, 1944.

38. R. Wong and T. Lang, On the points of inflection of Bessel functions of positive order, II, Canad. J. Math. 43 (1991), 628-651.

Department of Mathematics, University of South Florida, Tampa, FL 33620, U.S.A.

Department of Mathematics and Statistics, York University, North York, Ont. M3J 1P3, CANADA 